\title{
LA PROTECTION DE LA NATURE
}

\section{(A propos d'un livre récent)}

Depuis quelques années, on entend assez souvent parler de protection de la nature. Ces mots sont interprétés de manière différente et même, pour beaucoup, n'ont guère de signification. La grande masse du public reste indifférente ou incompréhensive vis-à-vis d'une question qui, acturellement, devrait se placer au premier rang des préoccupations de l'humanité. Dans un livre récemment publié (*), M. Roger Herm, Membre de l'Institut, Directeur du Museum et Vice-Président de l'Union internationale pour la Protection de la Nature, appelle l'attention sur ce sujet, de manière énergique et précise.

\section{Destruction de la Nature}

“ La Nature groupe, par définition, l'ensemble des êtres vivants, des lieux où ils vivent, des conditions qui régissent leurs rapports mutuels ». Au milieu de cet ensemble, l'Homme est apparu. L'humanité n'a pu s'installer, se développer qu'au prix d'une lutte incessante dont elle est sortie victorieuse. Mais cette victoire sur la nature a donné à l'homme la vanité, la tendance à dominer, l'habitude de détruire.

Le bilan de cette action destructrice est impressionnant. Pour ne parler que des animaux supérieurs, depuis une cinquantaine d'années, quarante espèces ont disparu de la surface du globe ; on estime que près de six cents sont en danger de disparaitre. On a pu écrire le nécrologe précis de certaines d'entre elles. Pour ne citer qu'un exemple, presque incroyable, la Colombe voyageuse (Ectopistes migratorius) qui, aux Etats-Unis, se déplaçait, dans le premier quart du $\mathrm{XIX}^{\mathbf{e}}$ siècle, en colonnes serrées d'une dizaine de kilomètres de long sur un kilomètre de large, est une espèce à jamais éteinte : le dernier individu est mort dans un jardin zoologique en I9I4. La liste est longue de semblables méfaits de l'homme et il convient de citer, à ce sujet. les sévères appréciations de M. Heim: " L'instinct de lutte, le désir de vaincre, ont donné naissance au besoin de détruire, ce qu'on appelle de nos jours la chasse sportive. La peur ancestrale est devenue plaisir du risque, appel du danger... En vérité. s’agit-il du

(*) Destruction et protection de la nature, far Roger HeIm. Armand Collin, éd., $22 \dot{4} \mathbf{p}$. 
goût du risque ? L'homme moderne se sert plus de sa prodigieuse technique que de son courage. La carabine est plus sûre que le javelot. Il chasse par un réflexe de vanité, pour la satisfaction de réaliser la preuve de son adresse et dans un sourd désir de triomphe ». Détruire un animal devenu rare, compromettre la survivance d'une espèce " est, sur le plan philosophique et scientifique, aussi grave peut-être que le meurtre d'un homme et aussi irréparable que la lacération d'un tableau de Raphaël ». Parfois, des animaux ont été pourchassés par instinct de lucre, tels les Chinchillas, petits rongeurs des Andes, dont la fourrure " a permis de vêtir des femmes élégantes dont il ne reste ni la silhouette, ni le souvenir, pas même celui de leurs manteaux d'hiver... tandis qu'irrémédiablement les Chinchillas ont disparu ). D'autres sont victimes de superstitions, comme le Rhinocéros de Java, à la corne duquel on attache de merveilleuses vertus curatives. Quelle qu'en soit la cause, le résultat de ces destructions inconsidérées est la présence, dans le monde animal, d'espèces réduites à un petit nombre d'individus. dont l'existence est plus ou moins précaire et qui sont de véritables reliques dont l'extermination est déjà prévisible.

S'il v a, du fait de l'homme, appauvrissement de la faune, il y a aussi amenuisement de la flore. En ce cas, l'action destructrice s'exerce indirectement par modification de la station ou directement par cueillette ou arrachage. Le dessèchement, le boisement d'une pelouse, l'exploitation à blanc étoc d'une forêt, l'incendie d'une lande, peuvent amener la disparition d'espèces qui ne trouvent plus les conditions qu'elles exigent. On sait combien le pâturage, dès qu'il est pratiqué avec quelque intensité, peut déterminer la raréfaction progressive et même la disparition de certaines plantes. Des espèces à fleurs ornementales peuvent disparaitre du fait de la cueillette : c'est le cas de cette belle Orchidée, le Sabot de Vénus (Cypripedium calceolus) dans des vallons boisés de l'Est, du trop recherché Edelweiss (I,eontopodium alpinum) dans les pelouses alpines, et de bien d'autres en des stations diverses. Le Bois gentil (Daphne mezereum) recherché à cause de sa floraison printanière, le Lauréole (Daphne laureola) estimé à cause de son feuillage persistant, ont été très raréfiés dans des forêts au voisinage des villes. Utilisé pour faire des infusions ou des liqueurs, le Génépy (Artemisia mutellina) est devenu rare dans les Alpes. L'arrachage de plantes par des horticulteurs ou des amateurs de jardins est encore plus funeste. Les botanistes herborisants même ont trop souvent une lourde responsabilité dans la destruction d'espèces rares: il en est “ dont l'esprit de collectionneur étouffe la conviction du naturaliste ».

Des exemples analogues nous sont fournis par certaines essences forestières. Le Sapin de Sicile (Abies nebrodensis) formait encore au $\mathrm{XVIII}^{\mathrm{e}}$ siècle des massifs de quelque étendue. L'exploitation et le pâturage l'ont fait disparaître à tel point qu'au début du $\mathrm{xx}^{\mathrm{e}}$ siècle on n'en connaissait qu'un seul pied, dans l'enceinte d'un couvent. En 
I927 a été découvert en Algérie, dans le Djurdjura, un petit boqueteau de Pin laricio qui, jusque là, avait échappé aux investigations: c'est une forme assez spéciale, que l'on a dénommée Pinus laricio var. mauretanica. Depuis est survenu un incendie et il ne subsiste plus que quelques arbres $(*)$.

Pour les plantes comme pour les animaux, il est des espèces qui, soit par leur rareté et leur stricte localisation, soit par leur isolement en une station aberrante, sont de véritables reliques. On cite le cas d'une Crucifère, l'Alysium pyrenaicum, dont il n'existe que quelques pieds sur un rocher des Pyrénées orientales; aux Iles Glénans croît une espèce spéciale de Narcisse. D'autres reliques sont des survivants maintenant isolés d'une végétation jadis installée à la faveur d'un climat différent. On explique ainsi la présence du Bouleau nain (Betula nana), espèce boréale, dans une tourbière de la Margeride, du Styrax (Styrax officinalis), espèce du bassin oriental de la Méditerranée, en une seule station provençale, la forêt de Montrieux (Var), de l'Alaterne (Rhammus alaternus), nettement méditerranéen, en un point des falaises de la Basse-Seine, près d'Elbeuf. Quelles qu'elles soient, ces reliques sont particulièrement exposées à être détruites ou raréfiées.

\section{Transformation de la Nature}

De manières diverses, l'homme détruit des animaux et des plantes, raréfie certaines espèces, en anéantit d'autres. S'opposer à cette action. permettre, le plus possible, le maintien de la faune et de la flore dans leurs caractères primitifs, c'est faire œuvre de protection de la nature. Une telle préoccupation laisse bien indifférente la grande masse du public, qui a tendance à ne voir dans ce souci de conservation d'un capital vivant que sentimentalité d'artiste ou manie de scientifique. Mais l'action destructrice de l'homme peut avoir des répercussions autrement graves qui compromettent le bien-être et même l'existence de l'humanité. A leur sujet, l'ignorance est funeste et l'indifférence coupable. La question de la protection de la nature doit prendre place dans les préoccupations de l'opinion mondiale.

\section{Rupture des équilibres naturels}

Le danger de l'action humaine sur la nature dérive essentiellement de ce que, par des interventions plus ou moins réfléchies,

(*) Grâce à des greffons que le dendrologue Dode a pu faire venir de Sicile, l'Abies nebrodensis est représenté par plusieurs pieds dans l'Arboretum des Barres. De même, des plants issus de graines de Pinus laricio var. mauretanica, envoyés en 1928, ont permis de constituer dans des arboretum de petits massifs de cette essence. Des espèces en danger de disparition totale dans la nature sont ainsi maintenues en culture. Le cas du Ginkao biloba, inexistant à l'état spontané, et qui n'a été conservé depuis des siècles que grâce à sa culture près des temples, est classique. 
l'homme provoque la rupture des équilibres naturels établis au cours des temps entre les êtres vivants rassemblés sur une même surface. $\mathrm{Du}$ fait des interréactions entre végétaux soumis aux mêmes condlitions de milieu, se constitue un groupement que l'on peut définir par son aspect ou par sa composition. On sait que, suivant le point de vue adopté, le groupement est une formation, où les espèces se révèlent par leur physionomie et leur importance numérique, ou bien une association, où les espèces, liées à des conditions d'existence uniformes, se mêlent en proportions déterminées. Mais aux plantes s'associent des animaux qui trouvent dans la station garnie de végétation leur habitat, leur biotope; les interréactions entre animaux et plantes concourent à expliquer l'ensemble du groupement d'êtres vivants, la biocénose.

C'est à propos de ces groupements, où dominent les végétaux ligneux, les forêts, que l'on peut observer les plus remarquables exemples d'équilibre naturel et aussi les plus redoutables cffets de la perturbation de ces équilibres. M. HEIm consacre à la description de la forêt, si diverse selon les climats, des pages que les forestiers liront avec plaisir. "Chez nous, la forêt attire la rêverie ou la quiète curiosité. Il y règne l'ambiance de chapelle. Simple, riante ou grave, elle séduit, elle berce... L'arbre qui la constitue nous est familier par son nom, son écorce. le contour de ses feuillles. Now savons quels oiseaux logent dans sa ramure, quelles herbes vivent à ses pieds, quels bolets forment son cortège... Nous avons ici recueilli l'Aspérule odorante parce que la futaie était de Hêtre. Nous parlons du Cène du Mélèze: nous savons que l'Oronge vient sous les Chênes... Nous avons forcé la nature à se plier à nos exigences et lui avons imposé de s'adapter aux catalogues ».

"Sous les tropiques, c'est autre chose. Rien de la douceur qu'apporte au regard la forêt de France; rien de son effet calmant ni de son ordonnance. Pas d'équilibre, pas de philosophie; ni sensibilité, ni amour. Il faut un effort pour la comprendre, même visuellement. Elle n'est pas faite pour nous; elle déroute d'abord. Pas de point de comparaison, pas d'échelle: ni dominant, ni dominé. Que devient la notion d'association végétale que notre sens anthropomorphique et le dogmatisme des phytosociologues ont assignée à nos groupements végéta11x, dont nous sommes en réalité à la fois les maitres et les esclaves ? La forêt tropicale n'est pas civilisée, elle est totalement sauvage. primitive. Comme l'indigène, elle est fragile. délicate. incompréhensible, déroutante et complexe dans sa physionomie... Vous êtes au milieu d'un monde multiple où tous les êtres sont différents et presque tous des inconnus, où tout semble hasard et mêlée. Ce ne sont pas les cinquante essences de toutes nos forêts francaises: ce sont mille, deux mille essences qui ne connaissent aucune loi de hiérarchie. La promiscuité n'a qu'une règle: la concurrence vitale. Les agencements insaisissables n'ont qu'une cause, le désordre ). 
Sur la forêt, l'homme exerce son action par l'exploitation plus ou moins intense, par l'incendie, par le pâturage du bétail. L'effet est bien divers suivant les contrées et les types de forêt. Dans la partie moyenne de l'Europe, grâce au climat et aux conditions d'évolution du sol, la forêt peut se reconstituer en devenant plus ou moins artificielle, mais aussi mieux adaptée aux besoins humains. Là où elle est détruite pour faire place à des prairies ou à des cultures, une sage tradition paysanne a conservé au sol sa fertilité. Le tableau est autre dans le bassin méditerranéen, de l'Espagne à l'Afriquıe du Nord et au Proche-Orient: à la suite du déboisement et de l'intensification du pâturage, l'érosion progresse dangereusement. Sous les tropiques, dans cette forêt que l'on a trop longtemps présentée comme plantureuse et d'une productivité indéfinie, alors qu'en réalité elle est fragile, les causes de destruction sont puissantes: c'est l'exploitation trop souvent brutale, le feu allumé en vue de cultures temporaires que l'on abandonne quand le sol est épuisé, des incendies inconsidérés. Parallèlement, le sol est particulièrement exposé à la dégradation, à la latérisation. La destruction de la forêt est irréversible: on marche vers la désertification et l'érosion. Le processus a été étudié, le danger a été maintes fois dénoncé, notamment par A. Chevalier et Aubreville en Afrique, par HumBERT à Madagascar. A la destruction de la forêt s'ajoutent, sous les tropiques, l'extension des monocultures industrielles, parfois l'augmentation du cheptel, toutes causes d'épuisement et d'érosion du sol.

C'est ainsi que, par rupture de l'équilibre naturel et impossibilité, en bien des régions, de rétablir un nouvel équilibre, on assiste en des pays divers, sur de vastes surfaces, à la ruine du sol. Soit du fait du climat, soit du faiti de l'homme, on estime qu'un quart environ de la superficie des terres du globe est actuellement improductif. En regard de cette stérilisation progressive, la population humaine s'accroît constamment: de I milliard d'individus au milieu du $\mathrm{XrX}^{\circ}$ siècle, elle a passé à I 600 millions en I900; elle est actuellement de 2400 millions, et on prévoit que dans un siècle elle sera de 4 milliards. A la rupture des équilibres naturels, se superpose la rupture de l'équilibre humain: plus l'homme détruit, moins il peut se nourrir, plus il se multiplie. "On comprend donc, écrit M. Heim, pourquoi le problème de la protection de la nature, longtemps enfermé dans un concept à la fois sentimental et scientifique, c'est-àdire associé à la sauvegarde d'espèces animales ou végétales spectaculaires, utiles ou rares, a peu à peu intégré des préoccupations économiques, dont la dégradation des sols est la plus essentielle... C'est le problème de l'avenir même de notre espèce, de l'Homo sapiens, qui est désormais posé. "Un vigoureux effort est nécessaire pour mettre fin aux procédés destructifs et régénérer, autant que possible. les zones appauvries et désertifiées. En Algérie, au Maroc, en Palestine, aux Etats-Unis, d'heureuses méthodes de restauration et 
conservation des sols ont été appliquées. Mais en trop de contrées, le désert restera l'état stable auquel a conduit l'action combinée du climat et de l'homme.

\section{Introduction d'espèces étrangères}

Il est encore des causes de rupture des équilibres naturels dont M. Негм a fait ressortir les conséquences parfois graves pour l'humanité.

C'est d'abord l'introduction, fortuite ou volontaire, en une contrée, d'animaux ou de.végétaux étrangers au pays. Assurément, nul ne songe à nier l'intérêt de la domestication de certains animaux et de la culture de certaines plantes exotiques. Des forestiers seraient mal venus, à l'heure actuelle, de formuler des objections à l'introduction en forêt du Douglas ou du Chêne rouge. Mais l'installation d'une espèce exotique dans une station nouvelle a des conséquences a priori imprévisibles: tantôt elle peut difficilement s'adapter, reste peu abondante ou disparait, tantôt elle devient envahissante au détriment d'autres. Partout, tant dans les régions tempérées que sous les tropiques, des plantes à faibles exigences se répandent et le tapis végétal tend à s'uniformiser. En Nouvelle-Zélande, pays à faune et flore assez pauvres, l'introduction de divers animaux et végétaux a déterminé de profonds changements; le Cerf, le Daim se sont multipliés et causent de sérieux dégâts, la Ronce et l'Ajonc sont devenus gênants pour les cultures. Le cas du Lapin en Australie est typique: 24 de ces animaux, débarqués en 1859 , sont à l'origine d'une prodigieuse pullulation contre laquelle les puissants moyens de défense employés sont restés inefficaces: les possibilités économiques de l'Australie sont actuellement réduites d'un quart du fait du lapin. Dans notre pays, nous subissons les conséquences de l'imprudente introduction du Poisson-chat dans nos eaux, et le Rat musqué, amené en Bohême en 1905, est devenu gênant près de certains étangs. Les exemples ne nous manquent pas de l'envahissement progressif de certaines plantes fortuitement introduites. Des graminées américaines gagnent du terrain dans des pelouses du Sud-Ouest; un arbrisseau des Etats-Unis, 1'Amorpha fruticosa, s'installe victorieusement dans les saulaies qui bordent le Bas-Rhône, tandis que, le long de ce fleuve comme le long du Rhin, la Verge d'Or du Canada (Solidago glabra) forme des taches d'une telle densité qu'elle compromet le développement des taillis d'Aunes et de Saules; l'Helodea canadensis encombre des rivières et des canaux. Dans nos cultures, l'Erigeron canadense est devenu une maulvaise herbe commune. De plus en plus, de telles intrusions d'êtres nouveaux dans la nature deviennent courantes. "Ainsi s'exagère le conflit qui oppose le nouvel ordre humain, créé par la civilisation, à l'ordre de la nature ». 


\section{Parasites et antiparasitaires}

Un élément intervient encore dans la rupture des équilibres naturels : ce sont les parasites, animaux ou végétaux, qui peuvent nuire à la prospérité d'une espèce et même la détruire. La présence de la mouche Tsé-tsé; véhiculant un trypanosome, rend inpossible l'existence de certaines espèces de bestiaux en Afrique; l'Endothia parasitica a pratiquement anéanti le Châtaignier (Castanea crenata) aux Etats-Unis; durant le premier quart de ce siècle, l'Ö̈dium du Chêne a fait considérablement régresser le Chêne tauzin dans le SudOuest. Avec les progrès de la civilisation et la facilité des transports, l'apparition de parasites nouveaux, parfois peu nocifs dans leur pays d'origine, mais pouvant être dangereux dans des biocénoses différentes, est un fait de plus en plus fréquent. En cultivant certaines espèces sur de grandes étendues, l'homme a multiplié le nombre et la nocuité des parasites qui lui imposent parfois de lourdes pertes. La lutte contre ces parasites est devenue une grave préoccupation et on a inventé de multiples substances antiparasitaires.

Mais, remarque avec sévérité $M$. HEIM, l'homme a tendance à ne considérer que ses intérêts immédiats, sans prévoir les lointaines répercussions de ses inventions. On répand des substances antiparasitaires, et cela parfois sur de grandes surfaces avec des moyens puissants; le ravageur disparait, mais avec lui bien des espèces qui interviennent dans l'équilibre naturel et peuvent avoir, du point de vue humain, un rôle utile, tels les insectes entomophages et les oiseaux. Ainsi se pose une question d'actualité qui préoccupe les milieux scientifiques et agricoles. Les forestiers, normalement désarmés vis-à-vis des invasions d'insectes en raison de l'étendue même et aussi de la topographie des forêts, ne peuvent que constater que des pullulations d'insectes qui semblent devoir compromettre gravement des massifs, s'atténuent et prennent fin rapidement par le seul effet de ia contre-attaque de parasites. Périodiquement, dans les hautes vallées des Alpes, des peuplements de Mélèze au feuillage roussi par la Pyrale grise (Steganoptycha pinicolana) semblent condamnés: au bout de 3 années, la situation est rétablie. L'arrêt complet, en 1927, grâce à la prolifération d'une bactérie parasite, d'une grave invasion de Dasychire (Dasychira pudibunda), qui en I925 et surtout en 1926, avait défeuillé des massifs de Hêtre dans la région d'Epinal, est un cas typique. Dans la lutte par des procédés chimiques contre des parasites, l'intervention humaine doit être mûrement réfléchie et on comprend que la Conférence internationale pour la protection de la nature, réunie à Lake Success en I949, ait instamment demandé la réglementation et le contrôle de l'emploi des antiparasitaires, ainsi que leur interdiction de principe dans les zones non cultivées. 


\section{TERRITOIRES PROTÉGÉS}

En présence de l'action destructrice ou dangereusement modificatrice de l'homme, la question s'est posée des mesures possibles pour arrêter cette action, maintenir les richesses naturelles et assurer leur exploitation sans aboutir à un appauvrissement. L'idée n'est pas nouvelle. On peut en trouver l'origine dans certaines restrictions au ḋoit de chasse, jadis édictées en des pays divers. Mais c'est en France, dans la forêt de Fontainebleau, que, sous la pression des artistes de Barbizon, on a vu pour la première fois s'affirmer la volonté de s'efforcer de maintenir un paysage végétal dans son état primitif, en le laissant indemne de toute intervention humaine. Les " réserves artistiques ) de la Forêt de Fontainebleau ont été organisées en 1853 .

Ultérieurement, en I872, était établi aux Etats-Unis le National Park de Yellowstone qui était défini comme " un domaine mis en - réserve pour les aspirations esthétiques, morales et sportives ». D’autre part, des congrès pour la protection des oiseaux se sont succédé à partir de 1872 et, en I933, une conférence pour la sauvegarde de la faune et de la flore d'Afrique a abouti à une convention internationale tendant à la protection d'un certain nombre d'espèces.

Ainsi a pris corps l'idée de surfaces protégées, de sanctuaires naturels, stuivant l'expression anglo-saxonne, établis, soit dans un but d'étude scientifique ou d'aspiration artistique, soit de récréation populaire ou d'éducation sociale. Suivant la conception qui est à leur origine, les surfaces protégées sont des parcs nationaux ou des réserves naturelles intégrales. Ces deux termes, souvent mal compris, doivent être bien définis.

“ Le Parc National est établi en vue de réserver pour la récréation dı public, sous contrôle rigoureux, des sanctuaires naturels dont la faune, la flore et les richesses du sol présentent un intérêt à la fois national, esthétique et éducatif ). La notion de parc national est donc éminemment touristique. Ainsi que le remarque M. Herm, " le danger de la formule réside dans les excès que la récréation pent faire naître ») il est nécessaire de réagir contre " un tourisme industriel et agressif $)$.

D'ailleurs, il y a bien des modalités dans le statut des parcs nationaux suivant leur situation et la mentalité des populations. Les parcs des Etats-Unis sont essentiellement touristiques, et certains se ressentent même d'une excessive affluence de visiteurs. Au contraire, en Suisse, dans le.Parc National de l'Engadine, les possibilités touristiques sont volontairement restreintes, les chemins sont rares et accessibles seulement à des personnes suffisamment entrainées à la marche en montagne, la circulation est sévèrement réglementée. Le but que l'on veut atteindre est la sauvegarde de richesses naturelles, leur étude par les naturalistes et la contemplation par un pu- 
blic qui, en Suisse, est parfaitement éduqué. En France, notre unique parc national, celui du Pelvoux, est de caractère touristique; mais l'interdiction de la chasse, la suppression du pâturage y assurent l'intégrité de la faune et de la flore, et les difficultés d'accès dans ce massif de haute montagne limitent l'affluence du public, ailleurs qu'en quelques points de la périphérie. Une conception différente a été appliquée en forêt de Fontainebleau: à côté de réserves biologiques destinées aux études des naturalistes et qui sont placées sous la sauvegarde du public, il est prévu des réserves artistiques, constituées notamment par de vieux peuplements, qui peuvent être mobiles et dont les limites peuvent varier lorsque la régénération ne peut être différée. Sans que le terme leur soit appliqué. il faut reconnaître que certaines forêts domaniales au relief accidenté, soumises à un traitement qui n'altère jamais profondément la physionomie des peuplements, sont, en fait, des parcs nationaux: telles sont la forêt de la Grande-Chartreuse, la forêt de la Sainte-Baume et d'autres encore.

Dans maintes autres forêts domaniales, les vieux peuplements constituent effectivement des réserves artistiques, surtout si on les prolonge au delà du terme normal d'exploitabilité, comme c'est le cas dans la forêt de la Joux pour le canton de la Glacière, dans la forêt de Bercé, pour une parcelle du canton des Clos. Un peu partout, dans nos forêts, des prescriptions spéciales ont été observées pour conserver le caractère de certains sites.

La création de réserves naturelles procède d'une idée toute différente de celle qui a présidé à l'établissement de parcs nationaux: il s'agit de maintenir aussi intacts que possible des groupements végétaux et animaux. A l'inverse des parcs nationaux, il est rigoureusement interdit au public d'y pénétrer et d'y causer la moindre perturbation: on laisse agir la nature. Seuls, avec toutes les précautions voulues, quelques naturalistes ou des personnalités administratives responsables sont autorisées à y circuler. L'expression la plus complète de cette conception est la réserve naturelle intégrale. Un exemple en existe à Madagascar pour une douzaine de massifs forestičs. En France une réserve intégrale, d'une étendue de I 5 ooo hectares, a été constituée en I928, dans la Basse Camargue, en une région de flore très spéciale et surtout d'un haut intérêt pour sa faune d'oiseaux migrateurs, par les soins de la Société nationale d'Acclimatation, avec le concours des compagnies industrielles rropriétaires du terrain. Il est aussi des réserves naturelles, à but défini, zoologique ou botanique, destinées à sauvegarder les types de végétation spontanée ou à protéger une espèce animale, réserves qui peuvent être d'étendue très limitée. La Suisse offre de nombreux exemples de pareilles réalisations, portant sur des tourbières, de petites surfaces de marais, des lieux de nidification de certains oiseaux. Quoique la stricte interdiction de circulation et même de pâturage n'ait pu y être imposée, on peut qualifier de réserve botanique le 
petit massif de Genèvrier thurifère (Juniperus thurifera), situé dans la vallée de la Durance au-dessus de Saint-Crépin (HautesAlpes) et appartenant, depuis I924, à l'Ecole des Eaux et Forêts. Les mesures prises pour la protection du Castor dans une partie des eaux du Rhône aboutissent, en fait, à la constitution d'une réserve zoologique. (Voir: Le castor rhodanien. par M. Cointat. Rev. For. Franç., avril I949, p. I9).

\section{Exploitation conservatrice de la Nature}

Il existe donc une technique de la protection de la nature, appliquée dans des pays de plus en plus nombreux. Mais ces territoires protégés ne peuvent être que des îlots épars. Dans trop de pays peut continuer, sur de vastes étendues, l'action qui aboutit à l'appauvrissement du sol et menace l'existence même de l'humanité. Le problème se pose de concilier sciencè, art et économie, il s'agit de " transformer l'exploitation destructive à court terme en consommation régulière, continue, permanente, c'est-à-dire régénératrice et constructive ). La chose est possible et M. Heim cite le cas des forêts de nos régions qui, abandonnées à elles-mêmes, peuvent évoluer de manière défavorable et qui " doivent leur stabilisation, la constance des milieux écologiques qu'elles recèlent, à la conduite judicieuse à laquelle les forestiers les soumettent ». Pour exploiter les sols sans les ruiner. il est nécessaire d'envisager, pour les pays neufs, un plan de mise en valeur dont on trouvera l'inspiration dans les méthodes traditionnelles suivies par les paysans européens attachés à leur sol depuis des siècles. En I949, à Lake Success, réunis pour la première fois sous l'égide de l’O.N.U.. des économistes, préoccupés d'utiliser au mieux les ressources du globe, des naturalistes, soucieux de protéger la faune et la flore, des éducateurs, inquiets de la disparition de richesses d'intérêt artistique et touristique, sont tombés d'accord pour admettre que des méthodes d'exploitation anarchiques et gaspilleuses doivent prendre fin.

Comment parvenir à ce but, que désirent atteindre des hommes de bonne volonté, de tendances différentes ? Evidemment, il existe, en des pays variés, des lois et textes réglementaires qui, de manières diverses, assurent la protection de la nature et refrènent les abus d'exploitation du sol. Mais lois et règlements n'ont d'efficacité que dans la mesure où le peuple les comprend et s'y soumet volontairement. La différence est grande entre la mentalité du peuple suisse, qui respecte scrupuleusement une législation assurant aussi bien la prudente exploitation des forêts que la protection des plantes alpines et des animaux, et la mentalité des peuples méditerranéens. "Devant une opinion insuffisamment alertée, frappée d'inconscience ou d'ignorance, inéduquée, la loi reste impuissante ». Faute d'une opinion nationale dûment avertie, on est exposé à voir des intérêts économiques temporaires annihiler les efforts faits dans 
un but scientifique ou artistique. Un exemple d'actualité en est fourni par l'extension de la culture du riz en Camargue qui, indirectement, menace l'équilibre biologique indispensable au maintien de la flore et de la faune.

Les faits démontrent donc amplement que la conservation des richesses naturelles et leur utilisation rationnelle dont dépend l'avenir de l'humanité, ne pourront être organisées qu'avec le plein consentement de la majorité. Elle ne peut exister sans l'éducation des peuples, éducation qui doit se faire de multiples façons, éminemment variables suivant les pays, par la presse, le livre, la parole, le cinéma, en agissant sur le grand public, mais plus spécialement sur la jeunesse. La tâche est immense. Cependant, des symptômes encourageants peuvent être enregistrés, parmi lesquels il faut citer, en première ligne, la création à Fontainebleau, en I948, de l'Union internationale pour la protection de la Nature, dont l'action s'exerce de manière de plus en plus marquée. M. Heim, après avoir fait ressortir tous les motifs d'inquiétude, conclut par une parole d'espoir: “ La cause profonde des peuples l'emportera quand même, en fin de compte, sur l'égoïsme des individus et l'inconscience des gouvernements ).

\section{Les Forestiers et la Protection de la Nature}

La question de la protection de la Nature, sous ses divers aspects, doit prendre une large place dans les préoccupations des forestiers. Par leurs attributions mêmes, ils sont des protecteurs de la nature; ils peuvent le devenir davantage. Envisagé de la manière la plus large, le problème consiste à maintenir le plus possible les équilibres naturcllement établis, tout en assurant la meilleure utilisation du sol pour les besoins humains. N'est-ce pas là le principe même de la sylviculture ? " Imitez la nature ", a conseillé PARADE, et les foresticrs français sont restés fidèles à ce précepte. Mais le forestier, s'il a le devoir d'assurer la productivité de la forêt, n'est pas uniquement dominé par des considérations utilitaires. Vivant en contact avec la nature, il est sensible à la beauté des sites et des peuplements; par sa formation neême il est ouvert à la compréhension des questions biologiques. Un peu partout et depuis longtemps, il est dans nos forêts des surfaces où les exploitations sont limitées; il est des séries de protection, des séries artistiques, des peuplements ou des arbres remarquables dont on cherche à prolonger l'existence. Du point de vue de la faune, l'organisation des réserves de chasse répond à un but analogue. Il est souhaitable de voir encore s'élargir cette conception et se manifester, en des cas variés, le souci de protection de la nature. Parfois se trouvent enclavées dans une forêt, une clairière marécageuse, une pelouse sèche. La tentation est fréquente d'essayer de boiser ces surfaces improductives: l'assèchement par drainage peut entraîner la disparition d'une plante rare, le 
boisement de la pelouse peut compromettre l'existence d'une autre. L'enlèvement systématique des arbres morts peut faire disparaître un champignon ou un insecte d'une exceptionnelle rareté. Sachons sacrifier" quelques ares qui, au surplus, ne peuvent souvent porter que de médiocres peuplements artificiels, sachons transiger avec les principes d'hygiène de la forêt pour conserver des raretés végétales ou animales.

De par leurs fonctions, les forestiers ont un rôle plus original: ils sont appelés à rétablir dans la nature l'ordre perturbé par les interventions humaines. 'C'est déjà le but atteint par la cessation d'exploitations inconsidérées, par le reboisement dans des forêts plus ou moins dévastées. C'est surtout le résultat de cette grande œuvre de la restauration des montagnes, abordée il y a quatre-vingtdix ans, intensifiée depuis I882.

Dans ces forêts nouvelles, telles qu'on peut en contempler dans les Cévennes dans le vaste massif de l'Aigoual, dans les Alpes en maints bassins torrentiels, la nature a repris ses droits: on assiste au rétablissement progressif de la flore et de la faune, en même temps que l'on voit disparaitre les inconvénients qu'entraînaient le déboisement et l'érosion. Plus récemment, les mêmes préoccupations ont conduit à entreprendre en Afrique du Nord la restauration des sols. Le forestier devient ainsi, non seulement le protecteur, mais le restaurateur de la nature, et le grand organisateur de la restat1ration des montagnes que fut Demontzey a bien fait ressortir la beauté de ce rôle: " Je ne sais pas, a-t-il écrit, de plus noble mission que celle d'aider la nature à reconstituer dans nos montagnes l'ordre qu'elle avait si bien établi et que seule l'imprévoyance de l'homme a changé en un véritable chaos ».

Ph. Guinier.'

\section{Congrès des Géomètres-Experts}

Une Exposition internationale des Travaux et Instruments des Géomètres aura lieu à la Sorbonne, à Paris, dụ 28 août au 5 septembre, à l'occasion du Congrès International des Géomètres-Experts. 